\title{
AUTOMORPHISM GROUPS OF THE IMPRIMITIVE COMPLEX REFLECTION GROUPS
}

\author{
JIAN-YI SHI ${ }^{凶}$ and LI WANG \\ (Received 15 August 2006; accepted 17 February 2007) \\ Communicated by E. A. O'Brien
}

\begin{abstract}
We describe the group of all reflection-preserving automorphisms of an imprimitive complex reflection group. We also study some properties of this automorphism group.
\end{abstract}

2000 Mathematics subject classification: primary 20F28; secondary 20F55, 20E36, $51 \mathrm{~F} 15$.

Keywords and phrases: reflections, imprimitive complex reflection groups, automorphism groups.

\section{Introduction}

Let $\mathbb{N}$ (respectively, $\mathbb{Z}, \mathbb{R}, \mathbb{C}$ ) be the set of all positive integers (respectively, integers, real numbers, complex numbers). For any $k \leq n$ in $\mathbb{N}$, we use the notation $[k, n]:=$ $\{k, k+1, \ldots, n\}$ and $[n]:=[1, n]$. Shephard and Todd classified all finite complex reflection groups (see [5]). There are two families of such groups: primitive and imprimitive. For any $m, p, n \in \mathbb{N}$ such that $p \mid m$ (read ' $p$ divides $m$ '), let $G(m, p, n)$ be the group consisting of all $n \times n$ monomial matrices whose nonzero entries $a_{1}, \ldots, a_{n}$ are the $m$ th roots of unity with $\left(\prod_{i=1}^{n} a_{i}\right)^{m / p}=1$. In [2], Cohen proved that any irreducible imprimitive reflection group is isomorphic to some $G(m, p, n)$ (see [2, Subsection 2.4]). We see that $G(m, p, n)$ is a Coxeter group if either $m \leq 2$ or $(p, n)=(m, 2)$.

By an automorphism $\phi$ of a reflection group $G$, we mean that $\phi$ is an automorphism of the group $G$ as an abstract group which sends any reflection of $G$ to a reflection. In the present paper, when we mention an automorphism of $G$, we always mean that $G$ is regarded as a reflection group. Denote by $\operatorname{Aut}(G)$ the group consisting of all automorphisms of $G$.

The aim of this paper is to describe the $\operatorname{group} \operatorname{Aut}(m, p, n):=\operatorname{Aut}(G(m, p, n))$.

Supported by the NSF of China, the SFUDP of China, Sino-Germany Centre (GZ310), PCSIRT and Shanghai Leading Academic Discipline Project (BH07).

(C) 2009 Australian Mathematical Society 1446-7887/2009 \$16.00 
Set

$$
\operatorname{Int}(m, p, n):=\left\{\tau_{g} \mid g \in G(m, p, n)\right\},
$$

where $\tau_{g}: x \mapsto g x g^{-1}$ is the inner automorphism of $G(m, p, n)$ determined by $g$. The structure of $\operatorname{Aut}(m, p, n)$ is well known in the case where $G(m, p, n)$ is a Coxeter group. More precisely, when $m \leq 2$,

$$
G(m, p, n) \in\left\{A_{h}, B_{k}, D_{l} \mid h \geq 1, k \geq 2, l \geq 4\right\}
$$

and

$$
\operatorname{Aut}(m, p, n) \cong \operatorname{Int}(m, p, n) \cdot \Gamma
$$

with $\Gamma$ the graph automorphism group of $G(m, p, n)$. On the other hand, we have $G(m, m, 2)=I_{2}(m)$, the dihedral group generated by two reflections $s_{\alpha}, s_{\beta}$, where $\alpha, \beta$ are two unitary vectors in a plane with inner product $(\alpha, \beta)=-\cos (\pi / m)$. Then $\operatorname{Aut}(m, m, 2)$ consists of all transformations which send $s_{\alpha}$ to any reflection $s_{\alpha^{\prime}}$ of $I_{2}(m)$ and $s_{\beta}$ to another reflection $s_{\beta^{\prime}}$ for some unitary vectors $\alpha^{\prime}, \beta^{\prime}$ satisfying $\left(\alpha^{\prime}, \beta^{\prime}\right)=\cos (k \pi / m)$ for some $1 \leq k<m$ with $\operatorname{gcd}(k, m)=1$ (see [3]).

So we need only consider the case of $m>2$ and $n>1$ and $(p, n) \neq(m, 2)$ for $\operatorname{Aut}(m, p, n)$ in this paper. Our results can be stated briefly as follows. Set

$$
\operatorname{Int}(m, 1, n)_{p}=\left\{\tau_{g}^{(p)} \mid g \in G(m, 1, n)\right\},
$$

where $\tau_{g}^{(p)}$ denotes the restriction of $\tau_{g}$ to $G(m, p, n)$. For any $k \in[m]$ with $\operatorname{gcd}(k, m)=1$, the transformation $\psi_{k}:\left(a_{i j}\right) \mapsto\left(a_{i j}^{k}\right)$ on $G(m, p, n)$ is in $\operatorname{Aut}(m, p, n)$ (see Lemma 3.5). Let

$$
\Psi(m):=\left\{\psi_{k} \mid k \in[m], \operatorname{gcd}(k, m)=1\right\} .
$$

We have

$$
\operatorname{Aut}(m, p, n)=\operatorname{Int}(m, 1, n)_{p} \rtimes \Psi(m)
$$

for $(m, p, n) \notin\{(3,3,3),(4,2,2)\}$. In particular,

$$
\operatorname{Aut}(m, p, n)=\operatorname{Int}(m, p, n) \rtimes \Psi(m)
$$

if $\operatorname{gcd}(p, n)=1$. We also determine the structure of $\operatorname{Aut}(m, p, n)$ in the exceptional cases (see Theorem 7.1). As a consequence, we obtain the order of $\operatorname{Aut}(m, p, n)$ in all cases (see Proposition 7.2).

The above description for the $\operatorname{group} \operatorname{Aut}(m, p, n)$ is also applicable to most cases of $G(m, p, n)$ being a Coxeter group (see Remark 7.3).

We also study some properties of $\operatorname{Aut}(m, p, n)$. Among others, we give an explicit description of its centre (see Propositions 7.4-7.6, Subsections 7.7 and 7.8 and Corollary 7.9).

This paper is organized as follows. In Section 2, we collect some concepts and results for later use. We study some general properties of $\operatorname{Aut}(m, p, n)$ in Section 3. In Sections 4-6, we describe $\operatorname{Aut}(m, p, n)$ explicitly in three cases: $p=1$ and $p=m$ and $p \in[2, m-1]$, respectively. In Section 7, we study some properties of $\operatorname{Aut}(m, p, n)$. 


\section{Preliminaries}

2.1. Let $V$ be a Hermitian space of dimension $n$. A reflection in $V$ is a unitary transformation of $V$ of finite order with exactly $n-1$ eigenvalues equal to 1 . A reflection group in $V$ is a finite group generated by reflections in $V$. A reflection group $G$ is called a real group or a Coxeter group if there is a $G$-invariant $\mathbb{R}$-subspace $V_{0}$ of $V$ such that the canonical map $\mathbb{C} \otimes_{\mathbb{R}} V_{0} \rightarrow V$ is bijective. Call $G$ a complex group otherwise (according to this definition, a real group is not complex).

2.2. A reflection group $G$ in $V$ is called imprimitive if $G$ acts on $V$ irreducibly and if $V$ is a direct sum $V=V_{1} \oplus V_{2} \oplus \cdots \oplus V_{t}$ of nontrivial proper subspaces $V_{i}(i \in[t])$ of $V$ such that $G$ permutes the set $\left\{V_{i} \mid i \in[t]\right\}$. In this situation, the family $\left\{V_{i} \mid i \in[t]\right\}$ is called a system of imprimitivity for $G$. Cohen [2] showed that any imprimitive complex reflection group is isomorphic to $G(m, p, n)$ for some $m, p, n \in \mathbb{N}$ with $p \mid m$ and $m>2$ and $n>1$ and $(p, n) \neq(m, 2)$; he also showed that $G(m, p, n)(p \mid m$ and $n \geq 2)$ has a unique system of imprimitivity if it is irreducible under the natural action on $\mathbb{C}^{n}$ and

$$
(m, p, n) \notin\{(2,1,2),(4,4,2),(3,3,3),(2,2,4)\} \quad \text { (see [2, Lemma 2.7]). }
$$

The group $G(1,1, n)(n \geq 2)$ is reducible and hence is not imprimitive.

In this paper, when the group $G(m, p, n)$ is mentioned, we always assume $p \mid m$ and $m>2$ and $n>1$ and $(p, n) \neq(m, 2)$ unless otherwise specified.

2.3. Any $w \in G(m, p, n)$ can be expressed in the form $w=\left[a_{1}, \ldots, a_{n} \mid \sigma\right]$ with some $\sigma \in \mathcal{S}_{n}$, where $\mathcal{S}_{n}$ is the symmetric group on the set [n] and $a_{i} \in \mathbb{Z}$ for $i \in[n]$, such that the entry of $w$ in the $(k,(k) \sigma)$-position is $\exp \left(\left(2 \pi a_{k} \sqrt{-1}\right) / m\right)$ for $k \in[n]$. We have $p \mid \sum_{k=1}^{n} a_{k}$.

An element $w=\left[a_{1}, \ldots, a_{n} \mid \sigma\right]$ of $G(m, p, n)$ is a reflection if one of the following conditions holds.

(1) We have $\sigma=(i, j)$, a transposition of $i$ and $j$ for some $i \neq j$ in $[n]$ and also $a_{i}+a_{j} \equiv 0$ and $a_{k} \equiv 0 \bmod m$ for $k \neq i, j$. In this case, denote $w$ by $s\left(i, j ; a_{i}\right)$ and call it a reflection of type I. Clearly, any reflection of type I has order 2 . We also have $s\left(i, j ; a_{i}\right)=s\left(j, i ;-a_{i}\right)$. All reflections of type I are contained in the subgroup $G(m, m, n)$ of $G(m, p, n)$;

(2) We have $\sigma=1$, and there exists $k \in[n]$ with $a_{k} \not \equiv 0$ and $a_{i} \equiv 0 \bmod m$ for all $i \in[n] \backslash\{k\}$. In this case, denote $w$ by $s\left(k ; a_{k}\right)$, and call it a reflection of type II. The reflection $s\left(k ; a_{k}\right)$ is a diagonal matrix with order $m / \operatorname{gcd}\left(m, a_{k}\right)$. Such reflections exist only when $p<m$.

By [1], we know that $G(m, p, n)$ has a generating set $S_{0}$ consisting of:

(i) $n+1$ reflections $s_{0}, s_{1}^{\prime}$ and $s_{i}$ for $i \in[n-1]$ if $p \in[2, m-1]$;

(ii) $n$ reflections $s_{0}$ and $s_{i}$ for $i \in[n-1]$ if $p=1$;

(iii) $n$ reflections $s_{1}^{\prime}$ and $s_{i}$ for $i \in[n-1]$ if $p=m$,

where $s_{0}=s(1 ; p)$ and $s_{1}^{\prime}=s(1,2 ;-1)$ and $s_{i}=s(i, i+1 ; 0)$. 
2.4. Let $G$ be a reflection group. Following Shi in [6, Subsection 1.9], a presentation of $G$ by generators and relations (or just a presentation of $G$ for short) is by definition a pair $(S, P)$, where:

(1) $S$ is a finite generating set for $G$ which consists of reflections, and $S$ has minimally possible cardinality with this property;

(2) $P$ is a finite set of relations on $S$, and any other relation on $S$ is a consequence of the relations in $P$.

We say that $S$ is a generating reflection set of $G$ if $S$ satisfies (1).

2.5. For $i \neq j$ and $i^{\prime} \neq j^{\prime}$ in $[n]$, and $k, k^{\prime}, l \in \mathbb{Z}$ with $m \nmid l$, denote $t=s(i, j ; k)$, $t^{\prime}=s\left(i^{\prime}, j^{\prime} ; k^{\prime}\right)$ and $s=s\left(i^{\prime} ; l\right)$. Then

$$
\begin{aligned}
& \begin{cases}t t^{\prime}=t^{\prime} t & \text { if }\{i, j\} \cap\left\{i^{\prime}, j^{\prime}\right\}=\emptyset, \\
t t^{\prime} t \cdots=t^{\prime} t t^{\prime} \cdots\left(m / \operatorname{gcd}\left(k-k^{\prime}, m\right) \text { factors on each side }\right) & \text { if }(i, j)=\left(i^{\prime}, j^{\prime}\right), \\
t t^{\prime} t \cdots=t^{\prime} t t^{\prime} \cdots\left(m / \operatorname{gcd}\left(k+k^{\prime}, m\right) \text { factors on each side }\right) & \text { if }(i, j)=\left(j^{\prime}, i^{\prime}\right), \\
t t^{\prime} t=t^{\prime} t t^{\prime} & \text { otherwise. }\end{cases} \\
& \qquad \begin{cases}t s=s t & \text { if } i^{\prime} \notin\{i, j\}, \\
s t s t=t s t s & \text { if } i^{\prime} \in\{i, j\} .\end{cases}
\end{aligned}
$$

From the above relations, we see that two noncommuting reflections $r, r^{\prime} \in$ $G(m, p, n)$ satisfy the relation $r r^{\prime} r r^{\prime}=r^{\prime} r r^{\prime} r$ if and only if either exactly one of $r, r^{\prime}$ has type II, or $r=s(i, j ; k)$ and $r^{\prime}=s\left(i, j ; k^{\prime}\right)$ for some $i \neq j$ in $[n]$ and some $k, k^{\prime} \in \mathbb{Z}$ with $m / \operatorname{gcd}\left(k-k^{\prime}, m\right)=4$. This fact will be useful in the subsequent discussion.

2.6. Denote by $o(s)$ the order of $s \in G(m, p, n)$. We have a presentation $\left(S_{0}, P_{0}\right)$ of the group $G(m, p, n)$, where $S_{0}$ is the generating reflection set as in Subsection 2.3 and $P_{0}$ is a relation set on $S_{0}$ given as follows (see [1]).

(1) When $p=1$, the set $P_{0}$ consists of the relations: $o\left(s_{0}\right)=m$ and $o\left(s_{i}\right)=2$ for $i \in[n-1] ; s_{i} s_{i+1} s_{i}=s_{i+1} s_{i} s_{i+1}$ for $i \in[n-2] ; s_{i} s_{j}=s_{j} s_{i}$ for $|i-j|>1$; $s_{0} s_{1} s_{0} s_{1}=s_{1} s_{0} s_{1} s_{0}$.

(2) When $p=m$, the set $P_{0}$ consists of the relations: $o\left(s_{1}^{\prime}\right)=o\left(s_{i}\right)=2$ for $i \in[n-1] ; s_{i} s_{i+1} s_{i}=s_{i+1} s_{i} s_{i+1}$ for $i \in[n-2] ; s_{i} s_{j}=s_{j} s_{i}$ for $|i-j|>1 ; s_{1}^{\prime} s_{i}=$ $s_{i} s_{1}^{\prime}$ for $i>2 ; s_{1}^{\prime} s_{2} s_{1}^{\prime}=s_{2} s_{1}^{\prime} s_{2} ; o\left(s_{1}^{\prime} s_{1}\right)=m ; s_{1}^{\prime} s_{1} s_{2} s_{1}^{\prime} s_{1} s_{2}=s_{2} s_{1}^{\prime} s_{1} s_{2} s_{1}^{\prime} s_{1}$.

(3) When $p \in[2, m-1]$, the set $P_{0}$ consists of the relations: $o\left(s_{1}^{\prime}\right)=o\left(s_{i}\right)=2$ for $i \in[n-1] ; o\left(s_{0}\right)=m / p ; s_{i} s_{i+1} s_{i}=s_{i+1} s_{i} s_{i+1}$ for $i \in[n-2] ; s_{i} s_{j}=s_{j} s_{i}$ for $|i-j|>1 ; \quad s_{1}^{\prime} s_{i}=s_{i} s_{1}^{\prime}$ for $i>2 ; \quad s_{1}^{\prime} s_{2} s_{1}^{\prime}=s_{2} s_{1}^{\prime} s_{2} ; o\left(s_{1}^{\prime} s_{1}\right)=m ; \quad s_{1}^{\prime} s_{1} s_{2} s_{1}^{\prime} s_{1} s_{2}=$ $s_{2} s_{1}^{\prime} s_{1} s_{2} s_{1}^{\prime} s_{1} ; s_{0} s_{1}^{\prime} s_{0} s_{1}^{\prime}=s_{1}^{\prime} s_{0} s_{1}^{\prime} s_{0} ; s_{0} s_{1} s_{0} s_{1}=s_{1} s_{0} s_{1} s_{0} ; s_{0} s_{1}^{\prime} s_{1}=s_{1}^{\prime} s_{1} s_{0} ;\left(s_{1}^{\prime} s_{1}\right)^{p-1}=$ $s_{0}^{-1} s_{1} s_{0} s_{1}^{\prime}$. 


\section{Automorphisms of a reflection group}

3.1. Denote by $\operatorname{Aut}(G)$ the automorphism group of $G$. The aim of this paper is to describe the automorphism group $\operatorname{Aut}(m, p, n):=\operatorname{Aut}(G(m, p, n))$ of the group $G(m, p, n)$.

Lemma 3.2 (See [7, Subsection 2.10 and Lemma 2.1], [8, Lemma 2.2]). Let $S$ be a generating reflection set of the group $G(m, p, n)$.

(1) If $p=1$, then $S$ consists of $n-1$ reflections of type I and one reflection of type II and order $m$.

(2) If $p=m$, then $S$ consists of $n$ reflections of type $I$.

(3) If $p \in[2, m-1]$, then $S$ consists of $n$ reflections of type I and one reflection of type II and order $m / p$.

Denote by $|X|$ the cardinality of a set $X$.

LEMMA 3.3. Let $S_{0}$ be the generating reflection set of $G(m, p, n)$ as in Subsection 2.3. Then for any $\eta \in \operatorname{Aut}(m, p, n)$, the image of $S_{0}$ under $\eta$ can be displayed as follows.

(1) When $p=1$, the $n$-tuple $\left(\eta\left(s_{0}\right), \eta\left(s_{1}\right), \ldots, \eta\left(s_{n-1}\right)\right)$ is equal to

$$
\left(s((1) \sigma ; k), s\left((1) \sigma,(2) \sigma ; k_{1}\right), \ldots, s\left((n-1) \sigma,(n) \sigma ; k_{n-1}\right)\right)
$$

for some $\sigma \in \mathcal{S}_{n}$ and $k, k_{1}, \ldots, k_{n-1} \in \mathbb{Z}$ with $k$ coprime to $m$.

(2) When $p=m$ and $(m, m, n) \neq(3,3,3)$, the $n$-tuple $\left(\eta\left(s_{1}^{\prime}\right), \eta\left(s_{1}\right), \ldots, \eta\left(s_{n-1}\right)\right)$ is equal to

$$
\left(s\left((1) \sigma,(2) \sigma ; k_{1}^{\prime}\right), s\left((1) \sigma,(2) \sigma ; k_{1}\right), \ldots, s\left((n-1) \sigma,(n) \sigma ; k_{n-1}\right)\right)
$$

for some $\sigma \in \mathcal{S}_{n}$ and $k_{1}^{\prime}, k_{1}, \ldots, k_{n-1} \in \mathbb{Z}$ with $\operatorname{gcd}\left(k_{1}-k_{1}^{\prime}, m\right)=1$.

(3) When $p \in[2, m-1]$ and $(m, p, n) \neq(4,2,2)$, the $(n+1)$-tuple $\left(\eta\left(s_{0}\right), \eta\left(s_{1}^{\prime}\right)\right.$, $\left.\eta\left(s_{1}\right), \ldots, \eta\left(s_{n-1}\right)\right)$ is equal to

$$
\left(s((1) \sigma ; p k), s\left((1) \sigma,(2) \sigma ; k_{1}^{\prime}\right), s\left((1) \sigma,(2) \sigma ; k_{1}\right), \ldots, s\left((n-1) \sigma,(n) \sigma ; k_{n-1}\right)\right)
$$

for some $\sigma \in \mathcal{S}_{n}$ and $k, k_{1}^{\prime}, k_{1}, \ldots, k_{n-1} \in \mathbb{Z}$ such that $\operatorname{gcd}\left(k_{1}-k_{1}^{\prime}, m\right)=1$ and $k_{1}-k_{1}^{\prime} \equiv k \bmod m / p($ hence, $\operatorname{gcd}(k, m / p)=1)$.

Proof. Let $\left(S_{0}, P_{0}\right)$ be the presentation of the group $G(m, p, n)$ as in Subsection 2.6. Then for any $\eta \in \operatorname{Aut}(m, p, n)$, the pair $\left(\eta\left(S_{0}\right), \eta\left(P_{0}\right)\right)$ is again a presentation of $G(m, p, n)$, where $\eta\left(P_{0}\right)$ is the relation set on $\eta\left(S_{0}\right)$ which is obtained from $P_{0}$ by substituting the elements of $S_{0}$ by the corresponding elements of $\eta\left(S_{0}\right)$.

(1) By the relation $o\left(\eta\left(s_{0}\right)\right)=o\left(s_{0}\right)=m>2$ in Subsection 2.6, we see that $\eta\left(s_{0}\right)$ is a reflection of type II. Now by Lemma 3.2(1), the images of all reflections in $S_{0} \backslash\left\{s_{0}\right\}$ must be of type I. We claim that there exist some permutation $h_{1}, h_{2}, \ldots, h_{n}$ 
of $1,2, \ldots, n$ and some $k, k_{1}, \ldots, k_{n-1} \in \mathbb{Z}$ with $\operatorname{gcd}(k, m)=1$ such that $\eta\left(s_{l}\right)=$ $s\left(h_{l}, h_{l+1} ; k_{l}\right)$ for $l \in[n-1]$ and $\eta\left(s_{0}\right)=s\left(h_{1} ; k\right)$. This can be seen by Subsection 2.5 and the relations:

(i) $\eta\left(s_{i}\right) \eta\left(s_{i+1}\right) \eta\left(s_{i}\right)=\eta\left(s_{i+1}\right) \eta\left(s_{i}\right) \eta\left(s_{i+1}\right)$ for $i \in[n-2]$;

(ii) $\eta\left(s_{i}\right) \eta\left(s_{j}\right)=\eta\left(s_{j}\right) \eta\left(s_{i}\right)$ for $i, j \in[0, n-1]$ with $|i-j|>1$;

(iii) $\eta\left(s_{0}\right) \eta\left(s_{1}\right) \eta\left(s_{0}\right) \eta\left(s_{1}\right)=\eta\left(s_{1}\right) \eta\left(s_{0}\right) \eta\left(s_{1}\right) \eta\left(s_{0}\right)$.

So (1) is proved by taking $\sigma \in \mathcal{S}_{n}$ with $(j) \sigma=h_{j}$ for $j \in[n]$.

(2) Recall that all reflections in $G(m, m, n)$ are of type I. By Subsection 2.6, we have the relations:

(i) $\eta\left(s_{i}\right) \eta\left(s_{i+1}\right) \eta\left(s_{i}\right)=\eta\left(s_{i+1}\right) \eta\left(s_{i}\right) \eta\left(s_{i+1}\right)$ for $i \in[n-2]$;

(ii) $\eta\left(s_{i}\right) \eta\left(s_{j}\right)=\eta\left(s_{j}\right) \eta\left(s_{i}\right)$ for $i, j \in[n-1]$ with $|i-j|>1$;

(iii) $o\left(\eta\left(s_{1}^{\prime}\right) \eta\left(s_{1}\right)\right)=m \geq 3$;

(iv) $\eta\left(s_{1}^{\prime}\right) \eta\left(s_{2}\right) \eta\left(s_{1}^{\prime}\right)=\eta\left(s_{2}\right) \eta\left(s_{1}^{\prime}\right) \eta\left(s_{2}\right)$;

(v) $\eta\left(s_{1}^{\prime}\right) \eta\left(s_{l}\right)=\eta\left(s_{l}\right) \eta\left(s_{1}^{\prime}\right)$ for $l \in[3, n-1]$.

Then by the assumption that $(m, m, n) \neq(3,3,3)$, there is a unique system of imprimitivity of $G(m, m, n)$ which is necessarily fixed by any automorphism (see Subsection 2.2). So we see by Subsection 2.5 that there exist some permutations $h_{1}, h_{2}, \ldots, h_{n}$ of $1,2, \ldots, n$ and some $k_{1}^{\prime}, k_{1}, \ldots, k_{n-1} \in \mathbb{Z}$ with $\operatorname{gcd}\left(k_{1}-k_{1}^{\prime}, m\right)=1$ such that $\eta\left(s_{i}\right)=s\left(h_{i}, h_{i+1} ; k_{i}\right)$ for $i \in[n-1]$ and $\eta\left(s_{1}^{\prime}\right)=$ $s\left(h_{1}, h_{2} ; k_{1}^{\prime}\right)$. So we obtain (2) by taking $\sigma \in \mathcal{S}_{n}$ with $(j) \sigma=h_{j}$ for $j \in[n]$.

(3) We claim that $\eta\left(s_{0}\right)$ is of type II. For otherwise, we would have $o\left(\eta\left(s_{0}\right)\right)=$ $m / p=2$ and exactly one reflection (say $t$ ) of type II in the set $\Delta=\left\{\eta\left(s_{1}^{\prime}\right), \eta\left(s_{i}\right) \mid i \in\right.$ $[n-1]\}$ by Lemma 3.2(3). If $n>2$, then there is also some $t^{\prime} \in \Delta \backslash\{t\}$ with $\left\{t, t^{\prime}\right\} \neq$ $\left\{\eta\left(s_{1}\right), \eta\left(s_{1}^{\prime}\right)\right\}$ and $t t^{\prime} \neq t^{\prime} t$ by the assumption of $n>2$. By Subsection 2.5, we would have $t t^{\prime} t t^{\prime}=t^{\prime} t t^{\prime} t$, which gives rise to a contradiction by Subsection 2.6(3). If $n=2$, then $S_{0}=\left\{s_{0}, s_{1}, s_{1}^{\prime}\right\}$. By Lemma 3.2(3) and the symmetry of $s_{1}, s_{1}^{\prime}$ in $S_{0}$, we may assume that $\eta\left(s_{0}\right)$ and $\eta\left(s_{1}^{\prime}\right)$ have type I, and $\eta\left(s_{1}\right)$ has type II without loss of generality. So $\eta\left(s_{1}\right)=s\left(h_{1} ; k\right)$ and $\eta\left(s_{0}\right)=s\left(h_{1}, h_{2} ; k_{1}\right)$ and $\eta\left(s_{1}^{\prime}\right)=s\left(h_{1}, h_{2} ; k_{1}^{\prime}\right)$ for some permutation $h_{1}, h_{2}$ of 1,2 and some $k_{1}, k_{1}^{\prime}, k \in \mathbb{Z}$. Hence $m=o\left(\eta\left(s_{1}\right) \eta\left(s_{1}^{\prime}\right)\right)=4$ by Subsections 2.5 and 2.6, which would imply $(m, p, n)=(4,2,2)$, contradicting our assumption. So the claim is proved.

Now $\eta\left(s_{0}\right)$ is of type II. Then all reflections in $\Delta$ are of type I by Lemma 3.2(3). By the same arguments as that in (1) and (2), we see by Subsections 2.5 and 2.6(3) that there are permutations $h_{1}, \ldots, h_{n}$ of $1, \ldots, n$ and some $k, k_{1}^{\prime}, k_{1}, \ldots, k_{n-1} \in \mathbb{Z}$ with $\operatorname{gcd}(k, m / p)=1$ and $\operatorname{gcd}\left(k_{1}-k_{1}^{\prime}, m\right)=1$ such that $\eta\left(s_{i}\right)=s\left(h_{i}, h_{i+1} ; k_{i}\right)$ for $i \in[n-1]$, that $\eta\left(s_{0}\right)=s\left(h_{1} ; p k\right)$ and that $\eta\left(s_{1}^{\prime}\right)=s\left(h_{1}, h_{2} ; k_{1}^{\prime}\right)$. Furthermore, by the relation

$$
\left(\eta\left(s_{1}^{\prime}\right) \eta\left(s_{1}\right)\right)^{p-1}=\eta\left(s_{0}\right)^{-1} \eta\left(s_{1}\right) \eta\left(s_{0}\right) \eta\left(s_{1}^{\prime}\right),
$$

we have $k_{1}-k_{1}^{\prime} \equiv k \bmod m / p$. Hence, we obtain (3) by taking $\sigma \in \mathcal{S}_{n}$ with $(i) \sigma=h_{i}$ for $i \in[n]$. 


\subsection{Set}

$$
\Phi(m):=\{i \in[m-1] \mid \operatorname{gcd}(i, m)=1\} .
$$

Then $\Phi(m)$ is a multiplicative group modulo $m$ of order $\phi(m)$, an Euler number. For any $k \in \Phi(m)$ and any $n \times n$ matrix $w=\left(a_{i j}\right)$, define $\psi_{k}(w)=\left(a_{i j}^{k}\right)$. In particular, when $w=\left[a_{1}, \ldots, a_{n} \mid \sigma\right] \in G(m, p, n)$, we have $\psi_{k}(w)=\left[k a_{1}, \ldots, k a_{n} \mid \sigma\right]$ $\in G(m, p, n)$. So $\psi_{k}$ can be regarded as a transformation on $G(m, p, n)$ (we adopt such a viewpoint from now on).

\section{LEMMA 3.5.}

(1) We have $\psi_{k} \in \operatorname{Aut}(m, p, n)$ for any $k \in \Phi(m)$.

(2) The set $\Psi(m):=\left\{\psi_{k} \mid k \in \Phi(m)\right\}$ forms a subgroup of $\operatorname{Aut}(m, p, n)$ of order $\phi(m)$.

Proof. Since $G(m, p, n)$ consists of monomial matrices, we have

$$
\psi_{k}(w y)=\psi_{k}(w) \psi_{k}(y) \quad \text { for any } w, y \in G(m, p, n) .
$$

By the condition $\operatorname{gcd}(k, m)=1$, there exists some $j \in \Phi(m)$ with $k j \equiv 1 \bmod m$. So $\psi_{k} \psi_{j}=\psi_{j} \psi_{k}=\psi_{1}$ is the identity transformation on $G(m, p, n)$. By the description of reflections in Subsection 2.3, we see that $\psi_{k}$ stabilizes the reflection set of $G(m, p, n)$. So $\psi_{k} \in \operatorname{Aut}(m, p, n)$. Hence, (1) is proved and (2) follows by noting that $\psi: k \mapsto \psi_{k}$ is an injective group homomorphism from $\Phi(m)$ to $\operatorname{Aut}(m, p, n)$ with the image $\Psi(m)$.

3.6. For any $g \in G(m, p, n)$, define

$$
\tau_{g}: G(m, p, n) \rightarrow G(m, p, n)
$$

by setting $\tau_{g}(x)=g x g^{-1}$ for any $x \in G(m, p, n)$. Then $\tau_{g}$ is an inner automorphism of $G(m, p, n)$ which stabilizes the reflection set of $G(m, p, n)$. Hence, $\tau_{g} \in$ $\operatorname{Aut}(m, p, n)$. Let

$$
\operatorname{Int}(m, p, n)=\left\{\tau_{g} \mid g \in G(m, p, n)\right\} .
$$

By a well-known result in group theory, we obtain the following result.

LEMMA 3.7. The subgroup $\operatorname{Int}(m, p, n)$ is a normal subgroup of $\operatorname{Aut}(m, p, n)$.

LEMMA 3.8. We have $\operatorname{Int}(m, p, n) \cap \Psi(m)=1$.

Proof. Assume $\tau \in \operatorname{Int}(m, p, n) \cap \Psi(m)$. Then there exist some $g=\left[a_{1}, \ldots, a_{n} \mid\right.$ $\sigma] \in G(m, p, n)$ and $k \in \Phi(m)$ with $\tau=\tau_{g}=\psi_{k}$. For any $x=\left[b_{1}, \ldots, b_{n} \mid \sigma^{\prime}\right]$ $\in G(m, p, n)$, we have $\tau_{g}(x)=\left[c_{1}, \ldots, c_{n} \mid \sigma \sigma^{\prime} \sigma^{-1}\right]$ for some $c_{1}, \ldots, c_{n} \in \mathbb{Z}$ and $\psi_{k}(x)=\left[k b_{1}, \ldots, k b_{n} \mid \sigma^{\prime}\right]$. The equation $\tau_{g}=\psi_{k}$ implies that $\sigma \sigma^{\prime} \sigma^{-1}=\sigma^{\prime}$ for any $\sigma^{\prime} \in \mathcal{S}_{n}$, that is, $\sigma$ is in the centre of $\mathcal{S}_{n}$. If $n>2$ then $\sigma=1$, hence $g$ is diagonal. Take any diagonal $x=\left[b_{1}, \ldots, b_{n} \mid 1\right] \in G(m, p, n)$ with $b_{1}, \ldots, b_{n}$ not all zero. We have

$$
\left[b_{1}, \ldots, b_{n} \mid 1\right]=\tau_{g}(x)=\psi_{k}(x)=\left[k b_{1}, \ldots, k b_{n} \mid 1\right] .
$$

This implies $k=1$ and hence $\tau=1$, as required. 
It remains to consider the case where $n=2$ and $\sigma=(12)$. Then $p<m$ by the assumption at the end of Subsection 2.2. The equation $\tau_{g}(x)=\psi_{k}(x)$ for any $x=\left[b_{1}, b_{2} \mid 1\right] \in G(m, p, n)$ amounts to the equation system $k b_{1} \equiv b_{2}$ and $k b_{2} \equiv$ $b_{1} \bmod m$ for any $b_{1}, b_{2} \in \mathbb{Z}$ with $p \mid\left(b_{1}+b_{2}\right)$. However, the latter does not always hold by observing the case of $b_{1}=0$ and $b_{2}=p$. So $\tau_{g} \neq \psi_{k}$ in this case.

So our result is proved.

3.9. For any $g \in G(m, 1, n)$, the inner automorphism $\tau_{g}$ of $G(m, 1, n)$ stabilizes the normal subgroup $G(m, p, n)$ of $G(m, 1, n)$, with the restriction $\tau_{g}^{(p)}:=\left.\tau_{g}\right|_{G(m, p, n)}$ being in $\operatorname{Aut}(m, p, n)$. We use the notation

$$
\operatorname{Int}(m, 1, n)_{p}=\left\{\tau_{g}^{(p)} \mid g \in G(m, 1, n)\right\},
$$

which forms a subgroup of $\operatorname{Aut}(m, p, n)$ normalized by $\Psi(m)$. We can show

$$
\operatorname{Int}(m, 1, n)_{p} \cap \Psi(m)=1
$$

by an argument similar to that for Lemma 3.8, hence

$$
\operatorname{Int}(m, 1, n)_{p} \Psi(m)=\operatorname{Int}(m, 1, n)_{p} \rtimes \Psi(m) .
$$

Use the notation $\iota=\tau_{s}^{(p)}$ with $s=s(1 ; 1) \in G(m, 1, n)$.

LEMMA 3.10. For any $x, y \in G(m, 1, n)$ and any divisor $p \in \mathbb{N}$ of $m$, we have $\tau_{x}^{(p)}=\tau_{y}^{(p)}$ if and only if $\tau_{x}=\tau_{y}$.

PROOF. We need only show that $\tau_{x}^{(p)}=\tau_{y}^{(p)}$ implies $\tau_{x}=\tau_{y}$. Now $\tau_{x}^{(p)}=\tau_{y}^{(p)}$ if and only if $\tau_{x}(g)=\tau_{y}(g)$ for any $g \in G(m, p, n)$. The latter holds if and only if $y^{-1} x$ lies in the centralizer $Z_{G(m, 1, n)}(G(m, p, n))$ of $G(m, p, n)$ in $G(m, 1, n)$. Thus, to show the equality $\tau_{x}=\tau_{y}$, we need only show that $Z_{G(m, 1, n)}(G(m, p, n))$ consists of scalar matrices.

Take any $z=\left[z_{1}, \ldots, z_{n} \mid \sigma\right] \in Z_{G(m, 1, n)}(G(m, p, n))$ with some $z_{1}, \ldots, z_{n} \in \mathbb{Z}$ and $\sigma \in \mathcal{S}_{n}$. By the equations

$$
\tau_{z}(s(i, i+1 ; 0))=s(i, i+1 ; 0) \quad \text { for all } i \in[n-1],
$$

we see that $\sigma$ lies in the centre of $\mathcal{S}_{n}$. We claim $\sigma=1$ (that is, $z$ is diagonal). It is obvious in the case $n>2$. If $n=2$ and $\sigma=(1,2)$, that is, $z=\left[z_{1}, z_{2} \mid(1,2)\right]$. Then the equations $\tau_{z}(s(1,2 ; k))=s(1,2 ; k)$ with $k=0,1$, imply that

$$
z_{1} \equiv z_{2} \bmod m \quad \text { and } \quad z_{1} \equiv z_{2}+2 \bmod m .
$$

This is impossible by our assumption of $m>2$. Hence, the claim is proved and so $z$ is diagonal. Then (4) further implies that

$$
z_{1} \equiv z_{2} \equiv \cdots \equiv z_{n} \bmod m
$$

So $z$ is a scalar matrix. Hence, our conclusion follows. 


\section{The group $\operatorname{Aut}(m, 1, n)$}

Theorem 4.1. We have $\operatorname{Aut}(m, 1, n)=\operatorname{Int}(m, 1, n) \rtimes \Psi(m)$.

PROOF. The group $\operatorname{Aut}(m, 1, n)$ has a normal subgroup $\operatorname{Int}(m, 1, n)$ and a subgroup $\Psi(m)$ by Lemmas 3.5 and 3.7. So $\operatorname{Aut}(m, 1, n)$ has a subgroup

$$
G:=\operatorname{Int}(m, 1, n) \Psi(m)=\operatorname{Int}(m, 1, n) \rtimes \Psi(m)
$$

by Lemma 3.8 .

Take any $\eta \in \operatorname{Aut}(m, 1, n)$. Then by (3.3.1), the image of the generating set $S_{0}$ of $G(m, 1, n)$ under $\eta$ is as follows:

$$
\begin{aligned}
& \left(\eta\left(s_{0}\right), \eta\left(s_{1}\right), \ldots, \eta\left(s_{n-1}\right)\right) \\
& \quad=\left(s((1) \sigma ; k), s\left((1) \sigma,(2) \sigma ; k_{1}\right), \ldots, s\left((n-1) \sigma,(n) \sigma ; k_{n-1}\right)\right)
\end{aligned}
$$

for some $\sigma \in \mathcal{S}_{n}$ and some integers $k, k_{1}, \ldots, k_{n-1}$ with $k$ coprime to $m$. Identify $\sigma$ with $[0, \ldots, 0 \mid \sigma] \in G(m, 1, n)$. Then

$$
\begin{aligned}
& \left(\left(\tau_{\sigma} \eta\right)\left(s_{0}\right),\left(\tau_{\sigma} \eta\right)\left(s_{1}\right), \ldots,\left(\tau_{\sigma} \eta\right)\left(s_{n-1}\right)\right) \\
& \quad=\left(s(1 ; k), s\left(1,2 ; k_{1}\right), \ldots, s\left(n-1, n ; k_{n-1}\right)\right) .
\end{aligned}
$$

There exists $w:=\left[p_{1}, \ldots, p_{n} \mid 1\right] \in G(m, 1, n)$ satisfying that $p_{j} \in[m]$ for $j \in[n]$, and $p_{i}-p_{i+1} \equiv-k_{i} \bmod m$ for $i \in[n-1]$. Then

$$
\left(\left(\tau_{w} \tau_{\sigma} \eta\right)\left(s_{0}\right),\left(\tau_{w} \tau_{\sigma} \eta\right)\left(s_{1}\right), \ldots,\left(\tau_{w} \tau_{\sigma} \eta\right)\left(s_{n-1}\right)\right)=\left(s(1 ; k), s_{1}, \ldots, s_{n-1}\right) .
$$

Since $\operatorname{gcd}(k, m)=1$, there exists a unique $c \in \Phi(m)$ with $k c \equiv 1 \bmod m$. Then $\psi_{c} \tau_{w} \tau_{\sigma} \eta=1$. Hence $\eta=\tau_{\sigma^{-1}} \tau_{w^{-1}} \psi_{c}^{-1} \in G$. So our equation is proved.

\section{The group $\operatorname{Aut}(m, m, n)$}

We describe $\operatorname{Aut}(m, m, n)$ in two cases: $(m, m, n)=(3,3,3)$ and $(m, m, n) \neq$ $(3,3,3)$.

5.1. Let $\left(S_{0}, P_{0}\right)$ be the presentation of $G(3,3,3)$ with $S_{0}=\left\{s_{1}^{\prime}, s_{1}, s_{2}\right\}$ and $P_{0}$ as in Subsections 2.3 and 2.6 (2). Define $\mu: S_{0} \rightarrow G(3,3,3)$ by setting $\mu\left(s_{1}^{\prime}\right)=$ $s(2,3 ;-1), \mu\left(s_{1}\right)=s_{1}$ and $\mu\left(s_{2}\right)=s_{2}$. We see that $\mu\left(S_{0}\right)$ is a generating reflection set of $G(3,3,3)$ and that all relations in $P_{0}$ remain valid when substituting $s$ by $\mu(s)$ for all $s \in S_{0}$. So $\mu$ can be extended to an automorphism of $G(3,3,3)$, still denoted by $\mu$.

Theorem 5.2. We have Aut $(3,3,3)=\left\langle\tau_{s_{1}}, \mu, \psi_{2} \cdot \iota\right\rangle$.

PROOF. This can be checked directly by GAP (see [4]). 
Theorem 5.3. Let $G_{1}:=\operatorname{Int}(m, m, n) \rtimes \Psi(m)$ and $G_{2}:=\operatorname{Int}(m, 1, n)_{m} \rtimes \Psi(m)$. Then $\operatorname{Aut}(m, m, n)=G_{2}$ for any $(m, m, n) \neq(3,3,3)$. In particular, if $\operatorname{gcd}(m, n)=1$, then $\operatorname{Aut}(m, m, n)=G_{1}$.

Proof. By Lemmas 3.5, 3.7, 3.8 and 3.10, we have $G_{1} \subseteq G_{2} \subseteq \operatorname{Aut}(m, m, n)$.

Take $\eta \in \operatorname{Aut}(m, m, n)$. Then by (3.3.2), the image $\eta\left(S_{0}\right)$ of $S_{0}$ under $\eta$ is as follows:

$$
\begin{aligned}
& \left(\eta\left(s_{1}^{\prime}\right), \eta\left(s_{1}\right), \ldots, \eta\left(s_{n-1}\right)\right) \\
& \quad=\left(s\left((1) \sigma,(2) \sigma ; k_{1}^{\prime}\right), s\left((1) \sigma,(2) \sigma ; k_{1}\right), \ldots, s\left((n-1) \sigma,(n) \sigma ; k_{n-1}\right)\right)
\end{aligned}
$$

for some $\sigma \in \mathcal{S}_{n}$ and $k_{1}^{\prime}, k_{1}, \ldots, k_{n-1} \in \mathbb{Z}$ with $\operatorname{gcd}\left(k_{1}-k_{1}^{\prime}, m\right)=1$. As before, identifying $\sigma$ with $[0, \ldots, 0 \mid \sigma] \in G(m, m, n)$, we obtain

$$
\begin{aligned}
& \left(\left(\tau_{\sigma} \eta\right)\left(s_{1}^{\prime}\right),\left(\tau_{\sigma} \eta\right)\left(s_{1}\right), \ldots,\left(\tau_{\sigma} \eta\right)\left(s_{n-1}\right)\right) \\
& \quad=\left(s\left(1,2 ; k_{1}^{\prime}\right), s\left(1,2 ; k_{1}\right), \ldots, s\left(n-1, n ; k_{n-1}\right)\right) .
\end{aligned}
$$

If $\operatorname{gcd}(m, n)=1$, then there exists a unique $w:=\left[p_{1}, \ldots, p_{n} \mid 1\right] \in G(m, m, n)$ satisfying that $p_{j} \in[m]$ for $j \in[n]$, and $p_{i}-p_{i+1} \equiv-k_{i} \bmod m$ for $i \in[n-1]$, and $m \mid \sum_{i=1}^{n} p_{i}$. Then$$
\left(\left(\tau_{w} \tau_{\sigma} \eta\right)\left(s_{1}^{\prime}\right),\left(\tau_{w} \tau_{\sigma} \eta\right)\left(s_{1}\right), \ldots,\left(\tau_{w} \tau_{\sigma} \eta\right)\left(s_{n-1}\right)\right)=\left(s\left(1,2 ; k_{1}^{\prime}-k_{1}\right), s_{1}, \ldots, s_{n-1}\right) .
$$

If $\operatorname{gcd}(m, n)>1$, then there exists $w^{\prime}:=\left[p_{1}^{\prime}, \ldots, p_{n}^{\prime} \mid 1\right] \in G(m, m, n)$ satisfying $p_{j}^{\prime} \in[m]$ for $j \in[n]$, and $p_{i}^{\prime}-p_{i+1}^{\prime} \equiv-k_{i} \bmod m$ for $i \in[2, n-1]$, and $m \mid \sum_{i=1}^{n} p_{i}^{\prime}$. We have

$$
\begin{aligned}
& \left(\left(\tau_{w^{\prime}} \tau_{\sigma} \eta\right)\left(s_{1}^{\prime}\right),\left(\tau_{w^{\prime}} \tau_{\sigma} \eta\right)\left(s_{1}\right), \ldots,\left(\tau_{w^{\prime}} \tau_{\sigma} \eta\right)\left(s_{n-1}\right)\right) \\
& \quad=\left(s\left(1,2 ; k_{1}^{\prime}+\left(p_{1}^{\prime}-p_{2}^{\prime}\right)\right), s\left(1,2 ; k_{1}+\left(p_{1}^{\prime}-p_{2}^{\prime}\right)\right), s_{2}, \ldots, s_{n-1}\right) .
\end{aligned}
$$

In this case,

$$
\begin{aligned}
& \left(\left(\iota^{p_{2}^{\prime}-p_{1}^{\prime}-k_{1}} \tau_{w^{\prime}} \tau_{\sigma} \eta\right)\left(s_{1}^{\prime}\right),\left({ }_{l}^{p_{2}^{\prime}-p_{1}^{\prime}-k_{1}} \tau_{w^{\prime}} \tau_{\sigma} \eta\right)\left(s_{1}\right), \ldots,\left(\iota^{p_{2}^{\prime}-p_{1}^{\prime}-k_{1}} \tau_{w^{\prime}} \tau_{\sigma} \eta\right)\left(s_{n-1}\right)\right) \\
& \quad=\left(s\left(1,2 ; k_{1}^{\prime}-k_{1}\right), s_{1}, s_{2}, \ldots, s_{n-1}\right) .
\end{aligned}
$$

In each of the cases (5.3.1) and (5.3.2), we have $\operatorname{gcd}\left(k_{1}^{\prime}-k_{1}, m\right)=1$, hence there exists a unique $c \in \Phi(m)$ such that $\left(k_{1}^{\prime}-k_{1}\right) c \equiv-1 \bmod m$. Then

$$
\eta= \begin{cases}\tau_{\sigma^{-1}} \tau_{w^{-1}} \psi_{c}^{-1} \in G_{1} & \text { if } \operatorname{gcd}(m, n)=1, \\ \tau_{\sigma^{-1}} \tau_{\left(w^{\prime}\right)^{-1}} \iota^{k_{1}+p_{1}^{\prime}-p_{2}^{\prime}} \psi_{c}^{-1} \in G_{2} & \text { if } \operatorname{gcd}(m, n)>1 .\end{cases}
$$

So our conclusion follows. 


\section{The group $\operatorname{Aut}(m, p, n)$ with $p \in[2, m-1]$}

In this section, we describe $\operatorname{Aut}(m, p, n)$ with $p \in[2, m-1]$. We deal with two cases: $(m, p, n)=(4,2,2)$ and $(m, p, n) \neq(4,2,2)$. Set $q=m / p$.

6.1. Let $\left(S_{0}, P_{0}\right)$ be the presentation of $G(4,2,2)$ with $S_{0}=\left\{s_{0}, s_{1}^{\prime}, s_{1}\right\}$ and $P_{0}$ as in Subsections 2.3 and 2.6(3). Define $v: S_{0} \rightarrow S_{0}$ by setting $v\left(s_{0}\right)=s_{1}$ and $v\left(s_{1}\right)=s_{0}$ and $v\left(s_{1}^{\prime}\right)=s_{1}^{\prime}$. We see that all relations in $P_{0}$ remain valid when substituting $s$ by $v(s)$ for all $s \in S_{0}$. So $v$ can be extended to an automorphism of $G(4,2,2)$ which is still denoted by $v$.

Theorem 6.2. We have Aut $(4,2,2)=\langle\iota, v\rangle$.

PROOF. This can be checked directly by GAP (see [4]).

Theorem 6.3. Let $G_{1}:=\operatorname{Int}(m, p, n) \rtimes \Psi(m)$ and $G_{2}:=\operatorname{Int}(m, 1, n)_{p} \rtimes \Psi(m)$. Then $\operatorname{Aut}(m, p, n)=G_{2}$ for any $(m, p, n) \neq(4,2,2)$. In particular, if $\operatorname{gcd}(p, n)=1$, then $\operatorname{Aut}(m, p, n)=G_{1}$.

Proof. By Lemmas 3.5, 3.7, 3.8 and 3.10, we have $G_{1} \subseteq G_{2} \subseteq \operatorname{Aut}(m, p, n)$.

Take $\eta \in \operatorname{Aut}(m, p, n)$. Then by (3.3.3), the image $\eta\left(S_{0}\right)$ of $S_{0}$ under $\eta$ is as follows:

$$
\begin{array}{r}
\left(\eta\left(s_{0}\right), \eta\left(s_{1}^{\prime}\right), \eta\left(s_{1}\right), \ldots, \eta\left(s_{n-1}\right)\right)=\left(s((1) \sigma ; p k), s\left((1) \sigma,(2) \sigma ; k_{1}^{\prime}\right),\right. \\
\left.s\left((1) \sigma,(2) \sigma ; k_{1}\right), \ldots, s\left((n-1) \sigma,(n) \sigma ; k_{n-1}\right)\right)
\end{array}
$$

for some $\sigma \in \mathcal{S}_{n}$ and some $k, k_{1}^{\prime}, k_{1}, \ldots, k_{n-1} \in \mathbb{Z}$ with $\operatorname{gcd}(k, q)=1$ and $\operatorname{gcd}\left(k_{1}-k_{1}^{\prime}, m\right)=1$ and $k_{1}-k_{1}^{\prime} \equiv k \bmod q$. By identifying $\sigma$ with $[0, \ldots, 0 \mid \sigma] \in$ $G(m, p, n)$, we obtain

$$
\begin{aligned}
& \left(\left(\tau_{\sigma} \eta\right)\left(s_{0}\right),\left(\tau_{\sigma} \eta\right)\left(s_{1}^{\prime}\right),\left(\tau_{\sigma} \eta\right)\left(s_{1}\right), \ldots,\left(\tau_{\sigma} \eta\right)\left(s_{n-1}\right)\right) \\
& \quad=\left(s(1 ; p k), s\left(1,2 ; k_{1}^{\prime}\right), s\left(1,2 ; k_{1}\right), \ldots, s\left(n-1, n ; k_{n-1}\right)\right) .
\end{aligned}
$$

When $\operatorname{gcd}(p, n)=1$, there exists $w:=\left[p_{1}, \ldots, p_{n} \mid 1\right] \in G(m, p, n)$ such that $p_{j} \in[m]$ for $j \in[n]$, and $p_{i}-p_{i+1} \equiv-k_{i} \bmod m$ for $i \in[n-1]$, and $p \mid \sum_{i=1}^{n} p_{i}$. Then

$$
\begin{aligned}
& \left(\left(\tau_{w} \tau_{\sigma} \eta\right)\left(s_{0}\right),\left(\tau_{w} \tau_{\sigma} \eta\right)\left(s_{1}^{\prime}\right),\left(\tau_{w} \tau_{\sigma} \eta\right)\left(s_{1}\right), \ldots,\left(\tau_{w} \tau_{\sigma} \eta\right)\left(s_{n-1}\right)\right) \\
& \quad=\left(s(1 ; p k), s\left(1,2 ; k_{1}^{\prime}-k_{1}\right), s_{1}, \ldots, s_{n-1}\right) .
\end{aligned}
$$

When $\operatorname{gcd}(p, n)>1$, there exists $w^{\prime}:=\left[p_{1}^{\prime}, \ldots, p_{n}^{\prime} \mid 1\right] \in G(m, p, n)$ such that $p_{j}^{\prime} \in[m]$ for $j \in[n]$, and $p_{i}^{\prime}-p_{i+1}^{\prime} \equiv-k_{i} \bmod m$ for $i \in[2, n-1]$, and $p \mid \sum_{i=1}^{n} p_{i}^{\prime}$. Then

$$
\begin{aligned}
& \left(\left(\tau_{w^{\prime}} \tau_{\sigma} \eta\right)\left(s_{0}\right),\left(\tau_{w^{\prime}} \tau_{\sigma} \eta\right)\left(s_{1}^{\prime}\right),\left(\tau_{w^{\prime}} \tau_{\sigma} \eta\right)\left(s_{1}\right), \ldots,\left(\tau_{w^{\prime}} \tau_{\sigma} \eta\right)\left(s_{n-1}\right)\right) \\
& \quad=\left(s(1 ; p k), s\left(1,2 ; k_{1}^{\prime}+\left(p_{1}^{\prime}-p_{2}^{\prime}\right)\right), s\left(1,2 ; k_{1}+\left(p_{1}^{\prime}-p_{2}^{\prime}\right)\right), s_{2}, \ldots, s_{n-1}\right) .
\end{aligned}
$$


In this case, let $\kappa=\iota^{p_{2}^{\prime}-p_{1}^{\prime}-k_{1}} \tau_{w^{\prime}} \tau_{\sigma} \eta$. Then

$\left(\kappa\left(s_{0}\right), \kappa\left(s_{1}^{\prime}\right), \kappa\left(s_{1}\right), \ldots, \kappa\left(s_{n-1}\right)\right)=\left(s(1 ; p k), s\left(1,2 ; k_{1}^{\prime}-k_{1}\right), s_{1}, s_{2}, \ldots, s_{n-1}\right)$.

In each of the cases (6.3.1) and (6.3.2), we have $\operatorname{gcd}\left(k_{1}^{\prime}-k_{1}, m\right)=1$, hence there exists a unique $c \in \Phi(m)$ with $\left(k_{1}-k_{1}^{\prime}\right) c \equiv 1 \bmod m$. Hence, $\left(k_{1}-k_{1}^{\prime}\right) c \equiv 1 \bmod q$ as $q \mid m$. Since $k_{1}-k_{1}^{\prime} \equiv k \bmod q$, we have $k c \equiv 1 \bmod q$. Then

$$
\eta= \begin{cases}\tau_{\sigma^{-1}} \tau_{w^{-1}} \psi_{c}^{-1} \in G_{1} & \text { if } \operatorname{gcd}(p, n)=1, \\ \tau_{\sigma^{-1}} \tau_{\left(w^{\prime}\right)^{-1}} c^{k_{1}+p_{1}^{\prime}-p_{2}^{\prime}} \psi_{c}^{-1} \in G_{2} & \text { if } \operatorname{gcd}(p, n)>1\end{cases}
$$

So our conclusion follows.

REMARK 6.4. Suppose that $(m, p, n) \neq(3,3,3),(4,2,2)$. We have

$$
|\operatorname{Int}(m, p, n)|=|G(m, p, n)| /|Z(m, p, n)|=n ! m^{n-1} / \operatorname{gcd}(n, p)
$$

and

$$
\left|\operatorname{Int}(m, 1, n)_{p}\right|=|\operatorname{Int}(m, 1, n)|=|G(m, 1, n)| /|Z(m, 1, n)|=n ! m^{n-1} ;
$$

the latter follows by Lemma 3.10, where $Z(m, p, n)$ is the centre of $G(m, p, n)$. Hence, $\operatorname{gcd}(p, n)=1$ if and only if $\operatorname{Int}(m, p, n)=\operatorname{Int}(m, 1, n)_{p}$. Let $G_{1}, G_{2}$ be given in Theorem 6.3 (respectively, Theorem 5.3). Then we see that $\operatorname{gcd}(p, n)=1$ if and only if $G_{1}=G_{2}$ if and only if $\iota \in G_{1}$.

\section{Some properties of $\operatorname{Aut}(m, p, n)$}

In this section, we shall study some properties of $\operatorname{Aut}(m, p, n)$. Theorem 7.1 summarizes the main results in Sections 4-6. Proposition 7.2 provides the order of $\operatorname{Aut}(m, p, n)$. In Propositions 7.4-7.6, we study the centre $Z(\operatorname{Aut}(m, p, n))$ of $\operatorname{Aut}(m, p, n)$ with $(m, p, n) \notin\{(3,3,3),(4,2,2)\}$. Then we study $\operatorname{Aut}(3,3,3)$ and $\operatorname{Aut}(4,2,2)$ in Subsections 7.7 and 7.8, respectively. Finally, the order of $Z(\operatorname{Aut}(m, p, n))$ is summarized in Corollary 7.9.

THEOREM 7.1. We have:

(1) $\operatorname{Aut}(m, p, n)=\operatorname{Int}(m, 1, n)_{p} \rtimes \Psi(m)$ if $(m, p, n) \notin\{(3,3,3),(4,2,2)\}$; in particular, $\operatorname{Aut}(m, p, n)=\operatorname{Int}(m, p, n) \rtimes \Psi(m)$ if $\operatorname{gcd}(p, n)=1$;

(2) $\operatorname{Aut}(3,3,3)=\left\langle\tau_{s_{1}}, \mu, \psi_{2} \cdot \iota\right\rangle$;

(3) $\operatorname{Aut}(4,2,2)=\langle\iota, v\rangle$.

Proposition 7.2. The order of the group $\operatorname{Aut}(m, p, n)$ is equal to $m^{n-1} \cdot n !$. $\phi(m)$ if $(m, p, n) \notin\{(3,3,3),(4,2,2)\}$, to 432 if $(m, p, n)=(3,3,3)$, and to 48 if $(m, p, n)=(4,2,2)$. 
PROOF. The result in the case of $(m, p, n) \in\{(3,3,3),(4,2,2)\}$ can be checked by GAP (see [4]). Now assume $(m, p, n) \notin\{(3,3,3),(4,2,2)\}$. By Remark 6.4, we have $\left|\operatorname{Int}(m, 1, n)_{p}\right|=n ! m^{n-1}$. This implies the result by Theorem 7.1(1).

REMARK 7.3. Recall the description of $\operatorname{Aut}(m, p, n)$ when $G(m, p, n)$ is a Coxeter group (see the introduction). We see that Theorem 7.1 and Proposition 7.2 also hold when $G(m, p, n)$ is a Coxeter group with $(m, p, n) \notin\{(2,2,4),(2,1,2),(1,1,2)\}$, where each of $G(2,2,4), G(2,1,2)$ has more than one system of imprimitivity (see Subsection 2.2). We see that

$$
\operatorname{Int}(2,1,4)_{2} \rtimes \Psi(2)=\operatorname{Int}(2,1,4)_{2}
$$

is a subgroup of $\operatorname{Aut}(2,2,4)$ of index 3 and, hence, $|\operatorname{Aut}(2,2,4)|=576$. We also see that

$$
\operatorname{Int}(2,1,2) \rtimes \Psi(2)=\operatorname{Int}(2,1,2)
$$

is a subgroup of $\operatorname{Aut}(2,1,2)$ of index 2 and, hence, $|\operatorname{Aut}(2,1,2)|=8$. Also,

$$
\operatorname{Aut}(1,1,2)=\operatorname{Int}(1,1,2)=1 \text {. }
$$

In Propositions 7.4-7.6, we assume $(m, p, n) \notin\{(3,3,3),(4,2,2)\}$.

Proposition 7.4. The group $Z(\operatorname{Aut}(m, p, n))$ is trivial when $n>2$.

PRoOF. Take $\eta \in Z(\operatorname{Aut}(m, p, n))$. Then $\eta \cdot \tau_{g}=\tau_{g} \cdot \eta$ for any $g \in S_{0}$ (see Subsection 2.3 for $\left.S_{0}\right)$. This implies that $\tau_{\eta(g) \cdot g^{-1}}$ is the identity automorphism of $G(m, p, n)$. Hence, $\eta(g) \cdot g^{-1} \in Z(m, p, n)$, that is,

$$
\eta(g)=\left[a_{g}, \ldots, a_{g} \mid 1\right] \cdot g \quad \text { for some } a_{g} \in[0, m-1] \text { with } n a_{g} \equiv 0 \bmod p \text {. }
$$

By Lemma 3.3, we see that both $g$ and $\eta(g)$ are reflections of $G(m, p, n)$ with the same type and order and hence the same eigenvalue multi-set. By the assumption $n>2$ and by comparing with the eigenvalue multi-sets on both sides of (7.4.1), we obtain $a_{g} \equiv 0 \bmod m$ for any $g \in S_{0}$. So $\eta=1$. The result follows.

Next we consider $Z(\operatorname{Aut}(m, p, 2))$. We deal with the cases of $p$ being odd and even separately.

Proposition 7.5. Assume that $p$ is odd. Then

$$
Z(\operatorname{Aut}(m, p, 2))= \begin{cases}\left\{1, \tau_{[0, m / 2 \mid 1]}, \tau_{s_{1}} \cdot \psi_{m-1}, \tau_{[0, m / 2 \mid(12)]} \cdot \psi_{m-1}\right\} & \text { if } m \text { is even } \\ \left\{1, \tau_{s_{1}} \cdot \psi_{m-1}\right\} & \text { if } m \text { is odd }\end{cases}
$$


PROOF. Since $p$ is assumed to be odd,

$$
\operatorname{Aut}(m, p, 2)=\operatorname{Int}(m, p, 2) \rtimes \Psi(m)
$$

by Theorem 7.1(1). Take $\eta \in Z(\operatorname{Aut}(m, p, 2))$. Then $\eta$ has a unique expression of the form $\tau_{g} \cdot \psi_{c}$ for some $g \in G(m, p, 2)$ and some $c \in \Phi(m)$.

We have $\eta \cdot \tau_{s}=\tau_{s} \cdot \eta$ for any $s \in S_{0}$. This implies that $\tau_{g^{-1} s^{-1} g \psi_{c}(s)}=1$, that is, $g^{-1} s^{-1} g \psi_{c}(s) \in Z(G(m, p, 2))$. Write $g=[a, b \mid \sigma]$ for some $\sigma \in \mathcal{S}_{2}$ and some $a, b \in[0, m-1]$ with $p \mid(a+b)$. Then by a direct computation with $s$ ranging over $S_{0}$, we obtain that modulo $m$ :

(i) $2 a \equiv 2 b$;

(ii) $2 c \equiv 2$ and $p c \equiv p$ if $\sigma=1$;

(iii) $2 c \equiv-2$ and $p c \equiv-p$ if $\sigma=(12)$.

By (i), we have $a \equiv b \bmod m$ if $m$ is odd, and $a \equiv b \bmod m / 2$ if $m$ is even. Let $H$ be the set $\left\{[0,0 \mid \sigma] \mid \sigma \in \mathcal{S}_{2}\right\}$ if $m$ is odd, and $\left\{[0,0 \mid \sigma],[0, m / 2 \mid \sigma] \mid \sigma \in \mathcal{S}_{2}\right\}$ if $m$ is even. Then the condition (i) implies that $\tau_{g} \in\left\{\tau_{s} \mid s \in H\right\}$. By (ii)-(iii), we get $c \equiv 1 \bmod m$ if $\sigma=1$, and $c \equiv-1 \bmod m$ if $\sigma=(12)$ by the assumption of $p$ being odd.

So far we have proved that $Z(\operatorname{Aut}(m, p, 2))$ is contained in

$$
H_{o}:=\left\{1, \tau_{s_{1}} \cdot \psi_{m-1}\right\}
$$

if $m$ is odd, and in

$$
H_{e}:=\left\{1, \tau_{s_{1}} \cdot \psi_{m-1}, \tau_{[0, m / 2 \mid 1]}, \tau_{[0, m / 2 \mid(12)]} \cdot \psi_{m-1}\right\}
$$

if $m$ is even. Since $H_{o}$ for $m$ odd (or $H_{e}$ for $m$ even) is obviously in $Z(\operatorname{Aut}(m, p, 2)$ ), our result follows.

Proposition 7.6. Assume that $p$ is even. Then

$$
Z(\operatorname{Aut}(m, p, 2))=\left\{1, \tau_{[0, m / 2 \mid 1]}^{(p)}, \tau_{s_{1}} \cdot \psi_{m-1}, \tau_{[0, m / 2 \mid(12)]}^{(p)} \cdot \psi_{m-1}\right\} .
$$

PROOF. Since $p$ is assumed to be even,

$$
\operatorname{Aut}(m, p, 2)=\operatorname{Int}(m, 1,2)_{p} \rtimes \Psi(m)
$$

by Theorem 7.1(2). Take $\eta \in Z(\operatorname{Aut}(m, p, 2))$. Then $\eta$ has a unique expression of the form $\tau_{g}^{(p)} \cdot \psi_{c}$ for some $g \in G(m, 1,2)$ and some $c \in \Phi(m)$.

We have $\eta \cdot \tau_{s}^{(p)}=\tau_{s}^{(p)} \cdot \eta$ for any $s \in S_{0}$, where $S_{0}$ is the generating set of $G(m, 1, n)$ as in Subsection 2.3. This implies that

$$
\tau_{g^{-1} s^{-1} g \psi_{c}(s)}^{(p)}=1, \quad \text { that is, } g^{-1} s^{-1} g \psi_{c}(s) \in Z_{G(m, 1,2)}(G(m, p, 2)) .
$$

Write $g=[a, b \mid \sigma]$ for some $\sigma \in \mathcal{S}_{2}$ and some $a, b \in[0, m-1]$. Then by a direct computation with $s$ ranging over $S_{0}$, we obtain that modulo $m$ : 
(i) $2 a \equiv 2 b$;

(ii) $c \equiv 1$ if $\sigma=1$;

(iii) $c \equiv-1$ if $\sigma=(12)$.

By (i), we have $a \equiv b \bmod m / 2$ since $m$ is even. So $Z(\operatorname{Aut}(m, p, 2))$ is contained in

$$
H:=\left\{1, \tau_{s_{1}} \cdot \psi_{m-1}, \tau_{[0, m / 2 \mid 1]}^{(p)}, \tau_{[0, m / 2 \mid(12)]}^{(p)} \cdot \psi_{m-1}\right\} .
$$

Since $H$ is obviously in $Z$ (Aut $(m, p, 2)$ ), our result follows.

Finally, we consider $\operatorname{Aut}(3,3,3)$ and $\operatorname{Aut}(4,2,2)$.

7.7. Recall the element $\mu \in \operatorname{Aut}(3,3,3)$ that was defined in Subsection 5.1. By Theorem 5.2, we have $\operatorname{Aut}(3,3,3)=\left\langle\tau_{s_{1}}, \psi_{2} \cdot \iota, \mu\right\rangle$ with $o\left(\tau_{s_{1}}\right)=o\left(\psi_{2} \cdot \iota\right)=2$ and $o(\mu)=3$. The group $\left\langle\tau_{s_{1}}, \psi_{2} \cdot \iota\right\rangle$ is isomorphic to the dihedral group of order 12; the elements $\tau_{s_{1}}$ and $\mu$ commute; while $\left\langle\psi_{2} \cdot \iota, \mu\right\rangle$ has order 48 , which can be presented as

$$
\left\langle\psi_{2} \cdot \iota, \mu \mid\left(\psi_{2} \cdot \iota\right)^{2}=\mu^{3}=\left(\left(\psi_{2} \cdot \iota\right) \mu\left(\psi_{2} \cdot \iota\right) \mu\left(\psi_{2} \cdot \iota\right) \mu^{-1}\right)^{2}=1\right\rangle .
$$

The centre of $\operatorname{Aut}(3,3,3)$ is trivial.

7.8. By Theorem 6.2, we have $\operatorname{Aut}(4,2,2)=\langle\iota, v\rangle$ with $v$ as defined in Subsection 6.1, which can be presented as

$$
\operatorname{Aut}(4,2,2)=\left\langle\iota, v \mid \iota^{4}=v^{2}=(\iota \cdot v)^{6}=1,(\iota \cdot v)^{3}=(v \cdot \iota)^{3}\right\rangle .
$$

The centre of Aut $(4,2,2)$ is a cycle group of order 2 and is generated by $(\iota \cdot v)^{3}$.

From Propositions 7.4-7.6 and Subsections 7.7 and 7.8, we obtain the following result immediately.

COROLlary 7.9. The cardinality of the group $Z(\operatorname{Aut}(m, p, n))$ is 1 if $n>2$ and $2 \cdot \operatorname{gcd}(m, 2) /\left(1+\delta_{4, m} \delta_{2, p}\right)$ if $n=2$, where $\delta_{x y}$ is 1 if $x=y$ and 0 otherwise.

\section{Acknowledgements}

We express our deep gratitude to the referees for their invaluable comments and to Bob Howlett who explained to us his results on $\operatorname{Aut}(m, p, n)$ when $G(m, p, n)$ is a Coxeter group.

\section{References}

[1] M. Broué, G. Malle and R. Rouquier, 'Complex reflection groups, braid groups, Hecke algebras', J. Reine Angew. Math. $\mathbf{5 0 0}$ (1998), 127-190.

[2] A. M. Cohen, 'Finite complex reflection groups', Ann. Sci. École Norm. Sup. (4) 9 (1976), 379-436.

[3] B. Franzsen and B. R. Howlett, 'Automorphisms of nearly finite Coxeter groups', Adv. Geom. 3 (2003), 301-338.

[4] A. Hulpke, Homomorphism Search, Module of GAP computer software package available at http://www.gap-system.org.

[5] G. C. Shephard and J. A. Todd, 'Finite unitary reflection groups', Canad. J. Math. 6 (1954), 274-304. 
[6] J. Y. Shi, 'Simple root systems and presentations for certain complex reflection groups', Comm. Algebra 33 (2005), 1765-1783.

[7] - 'Congruence classes of presentations for the complex reflection groups $G(m, 1, n)$ and G(m, $m, n)$ ', Indag. Math. (N.S.) 16(2) (2005), 267-288.

[8] - 'Congruence classes of presentations for the complex reflection groups $G(m, p, n)$ ', J. Algebra 284(1) (2005), 392-414.

JIAN-YI SHI, Department of Mathematics, East China Normal University, Shanghai, 200241, PR China

e-mail: jyshi11@yahoo.com

LI WANG, Mathematical and Science College, Shanghai Normal University, Shanghai, 200234, PR China

e-mail: wl.ecnu@163.com 